\title{
Iatrogenic Left Ventricular Outlet Obstruction with a Ventricular Septal Defect Occluder
}

\section{Chih-Jun Lai, Hsing-Hao Huang \\ Department of Anaesthesiology, National Taiwan University Hospital, Taipei, Taiwan}

\section{Background}

Transcatheter closure by using an occluder is a treatment option for postoperative residual ventricular septal defects (VSDs). However, ventricular septal occluder placement may cause life-threatening complications. Our case represented an iatrogenic left ventricular outlet obstruction caused by the placement of a VSD occluder. ${ }^{1}$

\section{Case Report}

An 11-month-old full-term girl was diagnosed with a double outlet right ventricle during pregnancy and received total correction with VSD repair. Residual VSDs with right heart volume overload and pulmonary hypertension were noted by cardiac echo. After receiving cardiac catheterization twice with VSD occluders following balloon atrial septostomy, heart failure with cyanosis was still existed. The patient was later transferred to our hospital for surgical treatment. After admission, cardiac echo indicated 2 residual VSDs, a large muscular trabecular type and subaortic inlet type; each VSD had deployed 2 occluders in the previous hospital. Severe left ventricular outlet obstruction with severe aortic regurgitation caused by occluder deployment was noted. The patients received cardiac surgery, including the re-do of 2 VSD patch repairs, atrial septal defect closure, occlude removals, and aortic valve and tricuspid valve plasty. The patient recovered following the surgery.

\section{Reference}

1. Iranian Journal of Pediatrics 2015 Apr 18; doi: 10.5812/ijp.386. 2.Journal of Interventional Cardiology 2014; 27:260-72.

\section{Discussion}

Postoperative residual VSDs are extremely common. VSD occluders are considered safe for managing the condition and avoiding second surgery. Several studies have reported life-threatening complications of VSD occluders, including residual shunt, cardiac dysrhythmias, and multiple conduction abnormalities. ${ }^{2}$ Our case presented an uncommon VSD-occluder complication involving left ventricular outlet tract obstruction. To prevent the complication, echocardiogram monitoring, such as transesophageal echo (TEE), during the procedure may help in early detection of the malpositioned occluders.

\section{Learning Points}

1.Dyspnea or other symptoms of heart failure may occur following the use of VSD occluders, and VSD occluder-related complications should be considered.

2. The TEE may aid clinicians determining hemodynamic status during the procedure.

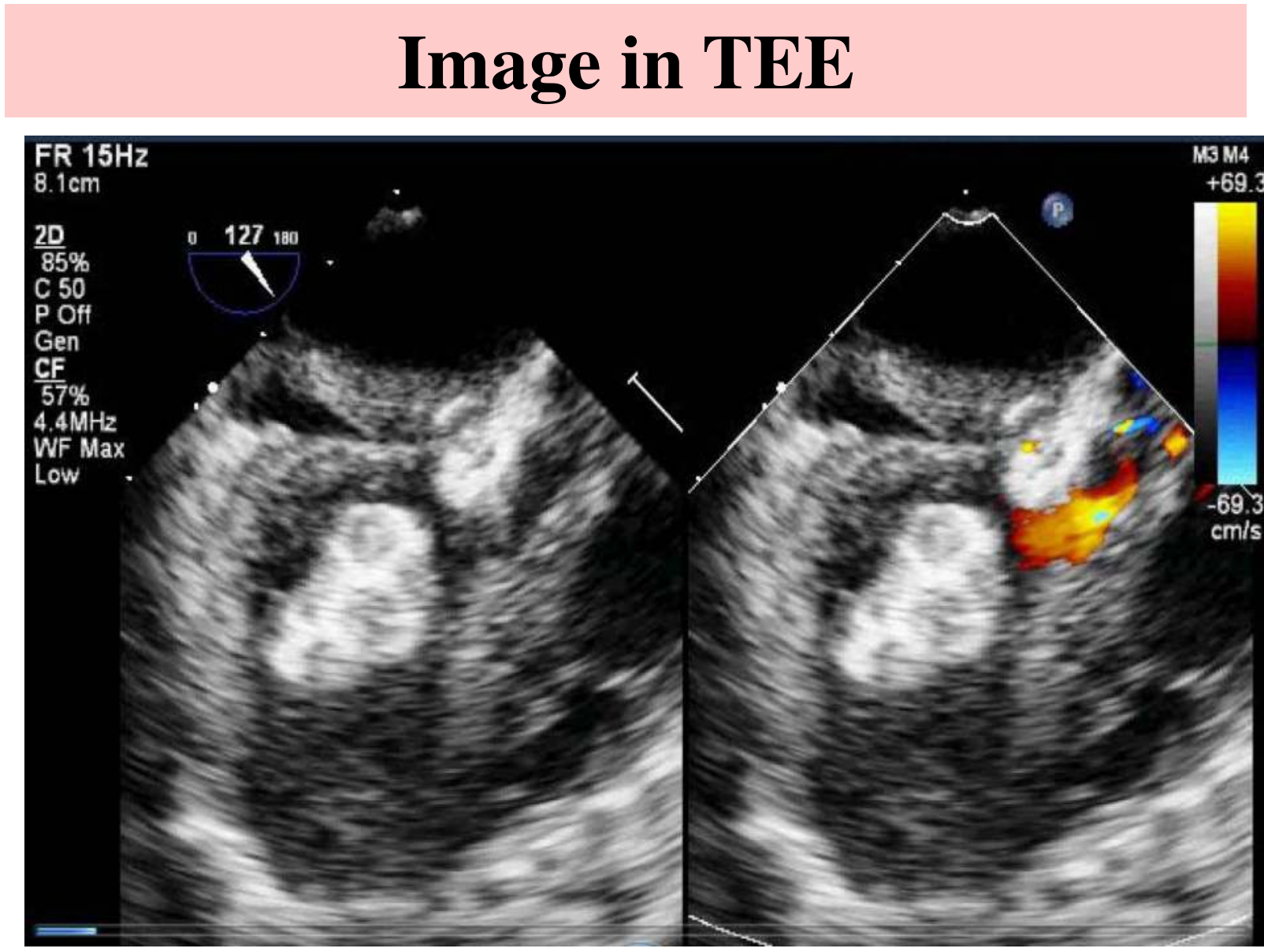

Severe left ventricular outlet obstruction with severe aortic regurgitation caused by occluder deployment 